\title{
Effect of Irreversibilities on the Performance of Thermoelectric Generator Investigated Using Exergy Analysis*
}

\author{
Chien-Chang WANG** and Chen-I HUNG** \\ **Department of Mechanical Engineering, National Cheng Kung University \\ No. 1, University Road, Tainan City 70101, Taiwan (ROC) \\ E-mail: cihung@mail.ncku.edu.tw
}

\begin{abstract}
For a practical thermoelectric generator (TEG) system, the performance is affected by many irreversible processes. This study evaluates the effects of multi-irreversibilities on TEG performance using exergy analysis. Based on the exergy analysis, two performance indexes, the energy efficiency and exergy efficiency, are used. A finite element scheme is employed to model the TEG system. The heat loss from the TEG to the environment and temperature-dependent material properties are considered. The results suggest that when the application of the small electrical current is considered, decreasing the hot-reservoir temperature or increasing the cold-reservoir temperature can improve the TEG exergy efficiency. Although heat loss slightly decreases the maximum energy and exergy efficiency, it can improve energy and exergy efficiencies for a case with large electrical current. On the other hand, when the Seebeck coefficient or thermal conductivity is temperature-dependent, both the maximum energy efficiency and exergy efficiency increase with increasing hot-reservoir temperature. However, the temperature-dependence of the electrical resistivity reduces the maximum exergy efficiency when the hot-reservoir temperature increases even though the maximum energy efficiency is increased.
\end{abstract}

Key words: Thermoelectric Generator (TEG), Exergy Analysis, Irreversibilities, Temperature-Dependent Properties, Finite Element Scheme

\section{Introduction}

The declining supply of fossil fuels has increased the urgency of developing new energy resources. The technology of thermoelectric waste heat recovery can be regarded as a renewable energy source ${ }^{(1)}$. Waste heat is usually recovered for further usage, such as space and water heating ${ }^{(2,3)}$, the improvement of energy recovery and system efficiency ${ }^{(4,5)}$, and the enhancement of chemical reactions ${ }^{(6,7)}$. Thermoelectric generators (TEGs) have advantages in the application of low-grade waste heat recovery due to their solid-state energy conversion mode ${ }^{(8)}$ and thus they have been discussed in many industrial fields ${ }^{(9)}$.

A TEG, which consists of many p-type and n-type semiconductors, can cleanly convert thermal energy to electrical energy based on the Seebeck effect ${ }^{(10)}$. TEGs have received a lot of attention due to their high reliability, lack of moving parts, and environmental friendliness ${ }^{(11,12)}$. Maneewan and Chindaruksa ${ }^{(13)}$ studied the performance of commercial thermoelectric modules using waste heat from a biomass dryer. The results showed that thermoelectric cooling modules have potential to be used as a TEG with waste heat from a

${ }^{*}$ Received 1 Oct., 2012 (No. 12-0412) [DOI: 10.1299/jtst.8.1]

Copyright () 2013 by JSME 
biomass dryer with a wall temperature of $180{ }^{\circ} \mathrm{C}$ to $200{ }^{\circ} \mathrm{C}$. Hsiao et al. ${ }^{(14)}$ built a one-dimensional theoretical model to simulate the TEG recovering waste heat from an automobile. Their study suggested that the thermoelectric module performed better on the exhaust pipe than on the radiator. Jang et al. ${ }^{(15)}$ investigated the effects of micro-TEG dimensions on TEG performance using finite element analysis. Their results showed that high efficiency was obtained when the length of the thermoelements was large. In addition, the power generated declined with the cross-sectional area of the thermoelements, whereas the efficiency showed the opposite trend. Wang et al. ${ }^{(16)}$ studied the performance of a TEG combined an air-cooling system designed using two-stage optimization. The results showed that using the two-stage optimization, the TEG output power density is enhanced by $88.70 \%$ compared to that without the optimal design though the heat sink efficiency is decreased by $20.93 \%$.

Practically, a TEG cannot work as a stand-alone device; it must be combined with heat exchangers to transfer heat to its heat reservoirs ${ }^{(17)}$. Regarding the TEG as a heat engine, an infinitely large surface area of heat exchangers or an infinite operating time is needed to achieve the reversible conditions. This corresponds to zero power output ${ }^{(18)}$. Therefore, in order to obtain a non-zero power output, finite-rate heat transfer between the heat engine and the environment is considered. This implies that there exist irreversibilities between the TEG and its heat reservoirs. There are also internal irreversibilities caused by the Joule heat and heat conduction through the thermoelectric elements between the hot and cold junctions ${ }^{(19)}$. Chen and $\mathrm{Wu}^{(19)}$ indicated that the effects of the external and internal irreversibilities on TEG performance must be considered simultaneously to obtain more significant results for TEGs.

As far as the practical thermoelectric system is considered, there exists an undesired irreversible process which also induces the irreversibilities. That is the heat loss from the thermoelectric devices to the ambient environment. The effect of heat loss on TEG performance has been concerned in recent research. Niu et al. ${ }^{(20)}$ constructed a TEG system combined with parallel-plate heat exchangers where a hot fluid and a cold fluid passed through the hot side and the cold side of the TEG, respectively. They found that the heat loss from the TEG to the environment increased significantly when the fluid inlet temperature at the hot side increased. Chen et al. ${ }^{(21)}$ built a CFD model to simulate the TEG performance. The results showed that TEG efficiency decreased when the heat transfer coefficient of the heat loss increased. However, their study did not consider the irreversibilities between the TEG and its heat reservoirs.

In the literature there are many papers proposing the importance of irreversibilities in TEG performance. However, few studies have comprehensively investigated the influence of external and internal irreversibilities using exergy analysis. Exergy is a quantitative assessment of the usefulness or quality of an energy quantity ${ }^{(22)}$. Exergy analysis provides more meaningful and useful information about efficiency and losses than does energy analysis ${ }^{(23)}$. A number of engineering applications, such as internal combustion engines ${ }^{(24)}$, solar collectors ${ }^{(25)}$, cogeneration power plants ${ }^{(26)}$, geothermal district heating systems ${ }^{(27)}$, and fuel cells ${ }^{(28)}$, have been investigated using exergy analysis. These studies demonstrated that exergy analysis is a useful tool for assessing energy-related systems. Consequently, the present study uses exergy analysis for evaluating the effects of various irreversibilities on TEG performance. The heat losses from thermoelectric elements to the environment are considered. Additionally, the effect of temperature-dependent material properties on TEG performance is taken into account. A finite element scheme is used to model the three-dimensional system and to predict the performance of a TEG. 


\section{Nomenclature}

$D$

$\vec{E}$

$E x_{i n}$

$E x_{\text {loss }}$

$E x_{\text {out }}$

I

$\vec{J}$

$k$

$L$

$P$

$\vec{q}$

$Q$

T

$U$

W

\section{Greek letters}

$\alpha$

$\phi$

$\eta$

$\eta_{c}$

$\eta_{e x}$

$\rho$

Subscripts

$c$

$H$

$h$

$L$

loss
Depth of TEG element, $\mathrm{mm}$

Electric field intensity vector, $\mathrm{V} \mathrm{m}^{-1}$

Exergy input of TEG, $\mathrm{W}$

Exergy loss of TEG, $\mathrm{W}$

Exergy output of TEG, $\mathrm{mW}$

Electric current, A

Electric current density vector, $\mathrm{A} \mathrm{m}^{-2}$

Thermal conductivity, $\mathrm{W} \mathrm{m}^{-1} \mathrm{~K}^{-1}$

Length of TEG element, $\mathrm{mm}$

Output power of TEG, $\mathrm{mW}$

Heat flux vector, $\mathrm{W} \mathrm{m}^{-2}$

Heat transfer rate, $\mathrm{W}$

Temperature, ${ }^{\circ} \mathrm{C}$

Overall heat transfer coefficient, $\mathrm{W} \mathrm{m}^{-2} \mathrm{~K}^{-1}$

Width of TEG element, mm

Seebeck coefficient, $\mathrm{V} \mathrm{K}^{-1}$

Electric scalar potential, $\mathrm{V}$

Energy efficiency, \%

Carnot cycle efficiency, $\%$

Exergy efficiency, \%

Electrical resistivity, $\Omega \mathrm{m}$

Cold side of the TEG element

Hot reservoir

Hot side of the TEG element

Cold reservoir

Heat loss from the lateral surfaces of the TEG elements

\section{Methodology}

\subsection{Physical model and assumptions}

A schematic of a TEG system that considers external irreversibilities is shown in Fig. 1 (a). In this system, the TEG operates between the hot and cold reservoirs at temperatures $T_{H}$ and $T_{L}$, respectively. The environment temperature is assumed to be $T_{L}$. There are heat exchangers between the heat reservoirs and the TEG. The heat transfer between the heat exchangers and the heat reservoirs is assumed to follow the Newton's law. The overall heat transfer coefficients of the hot-side and cold-side heat exchangers are expressed as $U_{H}$ and $U_{L}$, respectively. Additionally, the heat loss from the TEG to the environment also obeys the Newton's law, and is characterized by an overall heat transfer coefficient $U_{\text {loss }} . Q_{H}, Q_{L}$, and $Q_{\text {loss }}$ represent the heat input to the TEG from the hot reservoir, heat dissipated to the cold reservoir from the TEG, and heat loss from the TEG to the environment, respectively.

In this study, the TEG system is assumed to be steady-state. The TEG module is made up of identical thermoelectric couples. The electrodes are ignored and the contact resistances are neglected because the length of the thermoelectric element in this study is larger than $200 \mu \mathrm{m}{ }^{(29)}$. Based on the above assumptions, the computational domain includes only one thermoelectric couple comprising a p-type and an n-type elements. The geometry of the thermoelectric elements is shown in Fig. 1 (b). A thermoelectric element is specified by its depth $D$, width $W$, and length $L$. The lateral-side surfaces include all surfaces of the thermoelectric elements except the hot-side and cold-side surfaces. In the simulation, the 
p-type and n-type elements are connected electrically in series and thermally in parallel.

(a)
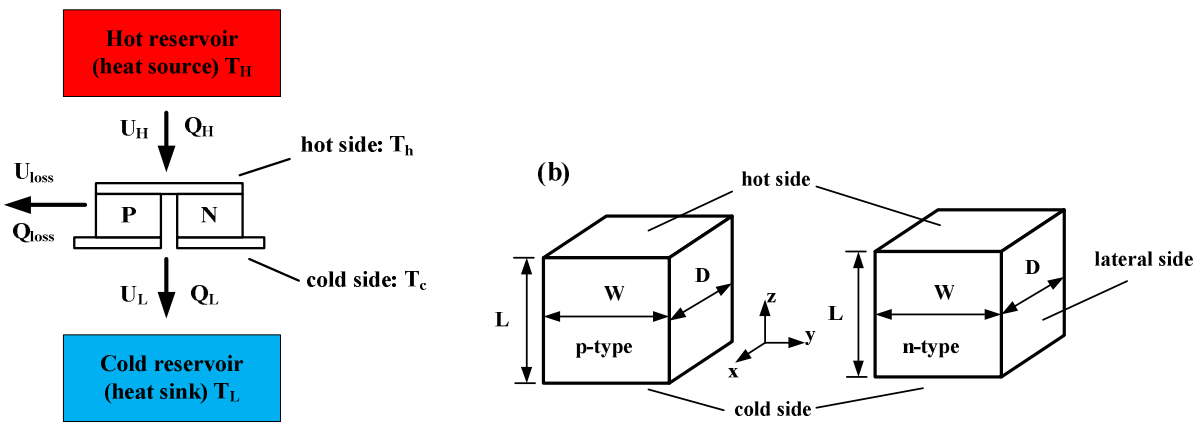

Fig. 1. Schematics of (a) irreversible TEG system and (b) thermoelectric couple geometry.

\subsection{Governing equations for TEG}

The thermoelectric effects caused by the thermoelectric elements can be expressed by the following constitutive equations ${ }^{(15)}$ :

$$
\begin{aligned}
& \vec{q}=\alpha T \vec{J}-k \nabla T \\
& \vec{J}=\frac{1}{\rho}(\vec{E}-\alpha \nabla T)
\end{aligned}
$$

where $\vec{q}, T$, and $\vec{J}$ represent the heat flux vector, absolute temperature, and current density vector, respectively; $\alpha, k$, and $\rho$ are the Seebeck coefficient, thermal conductivity, and electrical resistivity of a thermoelectric element, respectively. Electrical field $\vec{E}$ can be derived from electrical scalar potential $\phi$ :

$$
\vec{E}=-\nabla \phi
$$

The governing equations, including the thermal, electrical, and thermoelectric effects, are used to analyze the TEG system. Therefore, the steady-state conservation of energy and that of electric current are considered simultaneously ${ }^{(15)}$ :

$$
\begin{aligned}
& \nabla \cdot \vec{q}=\vec{J} \cdot \vec{E} \\
& \nabla \cdot \vec{J}=0
\end{aligned}
$$

The coupled governing equations for the electrical potential and temperature can be obtained by substituting Eqs. (1) (3) into Eq. (4) and Eq. (5):

$$
\begin{aligned}
& \nabla \cdot(\alpha T \vec{J})-\nabla \cdot(k \nabla T)=\vec{J} \cdot \vec{E} \\
& \nabla \cdot\left(\frac{1}{\rho} \nabla \phi\right)+\nabla \cdot\left(\frac{\alpha}{\rho} \nabla T\right)=0
\end{aligned}
$$

\subsection{Boundary conditions}

The boundary conditions for the present TEG model are as follows.

(1) The hot and cold sides of the thermoelectric elements are exposed to heat transfer with uniform heat transfer coefficients $U_{H}$ and $U_{L}$, respectively. This implies:

$-\left.k \frac{\partial T}{\partial z}\right|_{z=0}=U_{L}\left(T(z=0)-T_{L}\right)$

$-\left.k \frac{\partial T}{\partial z}\right|_{z=L}=U_{H}\left(T_{H}-T(z=L)\right)$

(2) When the heat loss is considered, a uniform heat transfer coefficient $U_{\text {loss }}$ is employed at the lateral-side surfaces of the thermoelectric elements:

$$
\begin{aligned}
& \left.k \frac{\partial T}{\partial x}\right|_{x=0}=U_{\text {loss }}\left(T(x=0)-T_{L}\right) \text { and }-\left.k \frac{\partial T}{\partial x}\right|_{x=D}=U_{\text {loss }}\left(T(x=D)-T_{L}\right) \\
& \left.k \frac{\partial T}{\partial y}\right|_{y=0}=U_{\text {loss }}\left(T(y=0)-T_{L}\right) \text { and }-\left.k \frac{\partial T}{\partial x}\right|_{y=D}=U_{\text {loss }}\left(T(y=W)-T_{L}\right)
\end{aligned}
$$


(3) The ground voltage is given as zero at the cold side of the p-type element.

\subsection{Exergy analysis for TEG}

In this study, two indexes are employed to evaluate TEG performance. One is the energy efficiency of TEG, expressed as:

$$
\eta=\frac{P}{Q_{H}}
$$

where $P$ and $Q_{H}$ represent the output power of the TEG and the heat input from the hot reservoir, respectively. The other performance index is the exergy efficiency of the TEG. It measures the quality level of the converted energy and is defined as ${ }^{(30)}$ :

$$
\eta_{\text {ex }}=\frac{E x_{\text {out }}}{E x_{\text {in }}}
$$

where $E x_{i n}$ and $E x_{\text {out }}$ represent the exergy input and exergy output of the TEG, respectively. The exergy input to the TEG is equal to the partial energy of heat input and is expressed as:

$$
E x_{i n}=\left(1-\frac{T_{L}}{T_{H}}\right) Q_{H}=\eta_{c} Q_{H}
$$

where $\eta_{c}$ is the Carnot cycle efficiency. The exergy output of the TEG is equal to the output power $P$. Therefore, the exergy efficiency can be rearranged as:

$$
\eta_{e x}=\frac{E x_{\text {out }}}{E x_{\text {in }}}=\frac{P}{\eta_{c} Q_{H}}=\frac{\eta}{\eta_{c}}
$$

That is, the exergy efficiency of the TEG can be expressed as the ratio of the energy efficiency to the Carnot cycle efficiency.

\subsection{Numerical method and validation}

The TEG model was built using commercial software (ANSYS v12.0.1). A finite element scheme based on the Galerkin method was used to discretize the governing equations ${ }^{(31)}$. Once the simulation was done, the TEG performance was calculated. In order to validate the proposed TEG model, some parameters and operating conditions were selected. According to the results presented by Maneewan and Chindaruksa ${ }^{(13)}$, the thermoelectric module TEC1-12708 was chosen as the geometry of the TEG in this study, as shown in Table 1.

Table 1 Parameters of the base case

\begin{tabular}{ll}
\hline $\begin{array}{l}\text { Parameters of the thermoelectric module } \\
\left(\text { TEC1-12708) }{ }^{(13)}\right.\end{array}$ & \\
\hline$W=1.4 \mathrm{~mm}$ & $U_{H}=6000 \mathrm{Wm}^{-2} \mathrm{~K}^{-1}$ \\
$D=1.4 \mathrm{~mm}$ & $U_{L}=250 \mathrm{Wm}^{-2} \mathrm{~K}^{-1}$ \\
$L=1.2 \mathrm{~mm}$ & $T_{H}=80{ }^{\circ} \mathrm{C}$ \\
No. of couples $=127$ & $T_{L}=24{ }^{\circ} \mathrm{C}$ \\
\hline
\end{tabular}

The material properties of the thermoelectric elements are given by ${ }^{(32)}$ :

$$
\begin{aligned}
& \alpha=\left(22224+930.6 T-0.9905 T^{2}\right) \times 10^{-9} \mathrm{VK}^{-1} \\
& \rho=\left(5112+163.4 T+0.6279 T^{2}\right) \times 10^{-10} \Omega \mathrm{m} \\
& k=\left(62605-277.7 T+0.4131 T^{2}\right) \times 10^{-4} \mathrm{Wm}^{-1} \mathrm{~K}^{-1}
\end{aligned}
$$

The constant material properties at $T=27{ }^{\circ} \mathrm{C}$ were initially used, that is, $\alpha=212.3 \times 10^{-6}$ $\mathrm{VK}^{-1}, \rho=1.106 \times 10^{-5} \Omega \mathrm{m}$, and $k=1.647 \mathrm{Wm}^{-1} \mathrm{~K}^{-1}$. The operating conditions of the base case are given in Table. $1^{(8)}$.

The case with heat loss was examined first. The heat transfer coefficient of heat loss was assumed to be $10 \mathrm{Wm}^{-2} \mathrm{~K}^{-1}{ }^{(9)}$. Because the temperature difference across the TEG significantly affects TEG performance, the temperature distribution of the thermoelectric element along the $\mathrm{z}$-direction was extracted to check grid independence. Three 
orthogonal-grid systems with 1,800, 5,850, and 14,400 cells, respectively, were individually tested. The numerical results are shown in Fig. 2 (a). Good grid independence was found, and thus, the grid system with 5,850 cells was chosen. Next, the numerical results were compared with the theoretical results. Because the theoretical method proposed by Gou et al. ${ }^{(8)}$ cannot deal with the heat loss from the lateral surfaces of the thermoelectric elements, the case without heat loss was examined. As shown in Fig. 2 (b), where $P, \eta$, and $\eta_{e x}$ are examined, the numerical results agree well with the theoretical results. The proposed model is thus suitable for analyzing TEG performance with multi-irreversibilities.

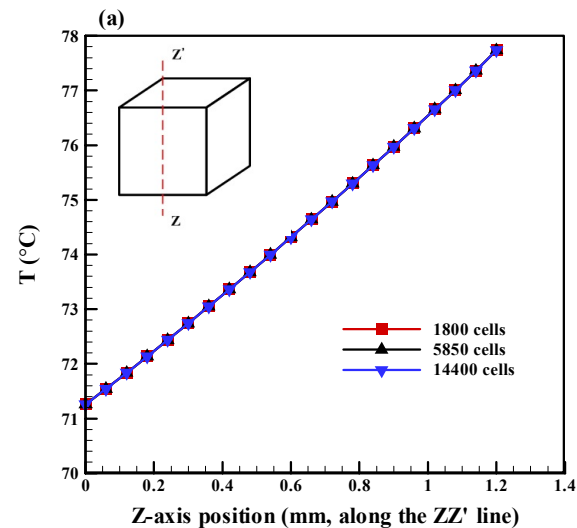

Fig. 2. (a) Grid independence check and theoretical results.

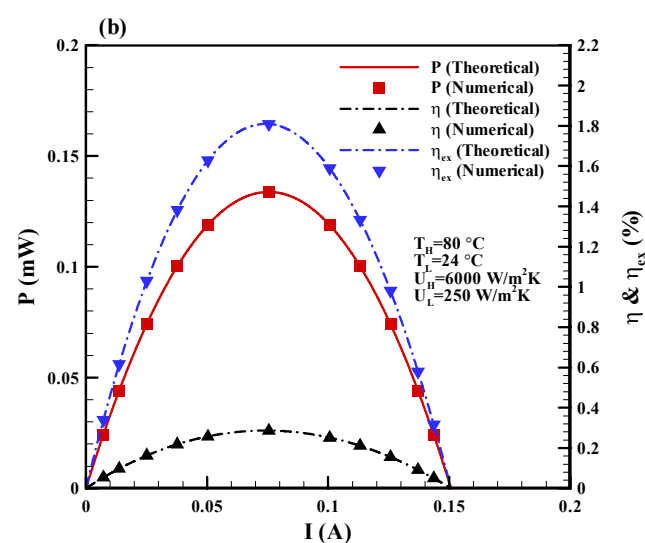

(b) TEG performance compared with the

\section{Results and discussion}

\subsection{Influence of heat loss}

The influence of heat loss from the thermoelectric elements to environment on TEG performance was investigated. The parameters and operating conditions of the base case shown in Table. 1 were used. Three kinds of heat transfer coefficient, $U_{\text {loss }}=0 \mathrm{Wm}^{-2} \mathrm{~K}^{-1}$ (i.e., no heat loss), $10 \mathrm{Wm}^{-2} \mathrm{~K}^{-1}$, and $20 \mathrm{Wm}^{-2} \mathrm{~K}^{-1}$, are considered. The results of exergy analysis versus electrical current are shown in Fig. 3. Figure 3 (a) shows that the curve of exergy out is parabolic whereas that of exergy input is linear and increases with increasing current. Due to the existence of inherent internal irreverisibilities, the order of magnitude of exergy output is smaller than that of exergy input. Additionally, the results indicate that both the exergy input and exergy output increase with increasing heat transfer coefficient of heat loss. This is due to the fact that more heat loss from the TEG means that more energy input is needed in order to satisfy the energy balance. Because the energy input is increased, the exergy input and exergy output increase. For exergy loss, which is defined as $E x_{\text {loss }}=E x_{\text {in }}-E x_{\text {out }}$, more heat loss results in more exergy loss, as shown in Fig. 3 (b). This finding indicates that compared to increase of exergy input, the increase of exergy output may be negligible when the heat loss is increased. As shown in Fig. 3 (b), the exergy loss is at its minimum when the electrical current approaches zero. However, this is not suitable for practical TEG applications.

The indexes of TEG performance, $\eta$ and $\eta_{e x}$, can be obtained from Fig. 3 (c). According to the definition of exergy efficiency, the percentage of useful energy that can be obtained from the TEG under multi-irreversibilities can be determined. As shown in Fig. 3 (c), the magnitude of $\eta_{e x}$ is higher than that of $\eta$. Furthermore, the developments of $\eta$ and $\eta_{e x}$ have similar trends when the heat transfer coefficient of heat loss is varied. That is, the existence of heat loss leads to slight reductions in both the maximum $\eta$ and maximum $\eta_{e x}$. Additionally, the distributions of $\eta$ and $\eta_{e x}$ both slightly shift in the positive-current direction. Therefore, the case without heat loss exhibits higher $\eta$ and 
$\eta_{e x}$ values than those of that with heat loss for a small electrical current whereas the opposite is true for a large electrical current.
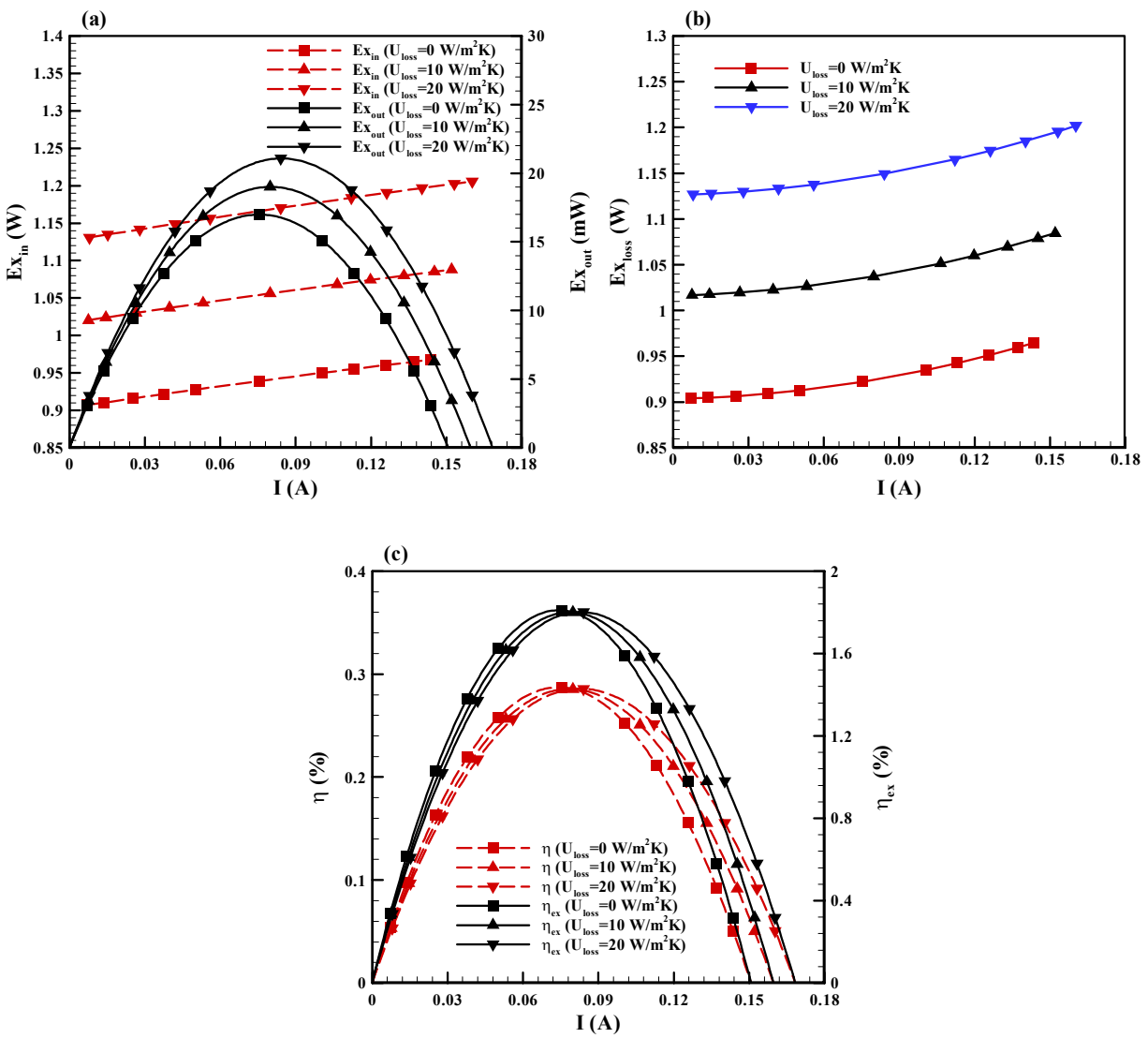

Fig. 3. Effects of heat loss on TEG performance in terms of (a) exergy input and exergy output, (b) exergy loss, and (c) energy efficiency and exergy efficiency.

\subsection{Influence of external irreversibilities}

In order to investigate the effects of external irreversibilities on TEG performance, four cases corresponding to different heat transfer coefficients at the hot side and cold side of the TEG were used. They are summarized in Table. 2. Heat transfer coefficients of 250 $\mathrm{Wm}^{-2} \mathrm{~K}^{-1}$ and $6000 \mathrm{Wm}^{-2} \mathrm{~K}^{-1}$ can be regarded as an air-cooling (heating) system and a water-cooling (heating) system, respectively ${ }^{(8)}$. The other operating conditions for each case are fixed. The TEG performance for the four cases is shown in Fig 4. The results reveal that, in general, the energy and exergy efficiencies improve with increasing heat transfer coefficient. This is due to larger heat transfer coefficients letting the hot-side and cold-side temperatures of the TEG be closer to the hot-reservoir and cold-reservoir temperatures, respectively. Consequently, the temperature difference across the TEG is increased and thus the TEG performance is improved. The range of electrical current is also expanded when the heat transfer coefficients increase. It should be noted that comparing to the case 1 , a noticeable enhancement in TEG performance is obtained when the heat transfer coefficients of both sides of the TEG are increased. As shown in Fig 4, a maximum $\eta_{e x}$ of $9.77 \%$ can be obtained by Case 4 but only $0.97 \%$ can be obtained by Case 1 .

Table 2 Operating conditions of four cases with different heat transfer coefficients

\begin{tabular}{llllll}
\hline Case & $U_{H}$ & $U_{L}$ & $T_{H}$ & $T_{L}$ & $U_{\text {loss }}$ \\
\hline 1 & $250 \mathrm{Wm}^{-2} \mathrm{~K}^{-1}$ & $250 \mathrm{Wm}^{-2} \mathrm{~K}^{-1}$ & $80{ }^{\circ} \mathrm{C}$ & $24{ }^{\circ} \mathrm{C}$ & $10 \mathrm{Wm}^{-2} \mathrm{~K}^{-1}$ \\
2 & $6000 \mathrm{Wm}^{-2} \mathrm{~K}^{-1}$ & $250 \mathrm{Wm}^{-2} \mathrm{~K}^{-1}$ & & & \\
3 & $250 \mathrm{Wm}^{-2} \mathrm{~K}^{-1}$ & $6000 \mathrm{Wm}^{-2} \mathrm{~K}^{-1}$ & & & \\
4 & $6000 \mathrm{Wm}^{-2} \mathrm{~K}^{-1}$ & $6000 \mathrm{Wm}^{-2} \mathrm{~K}^{-1}$ & & & \\
\hline
\end{tabular}




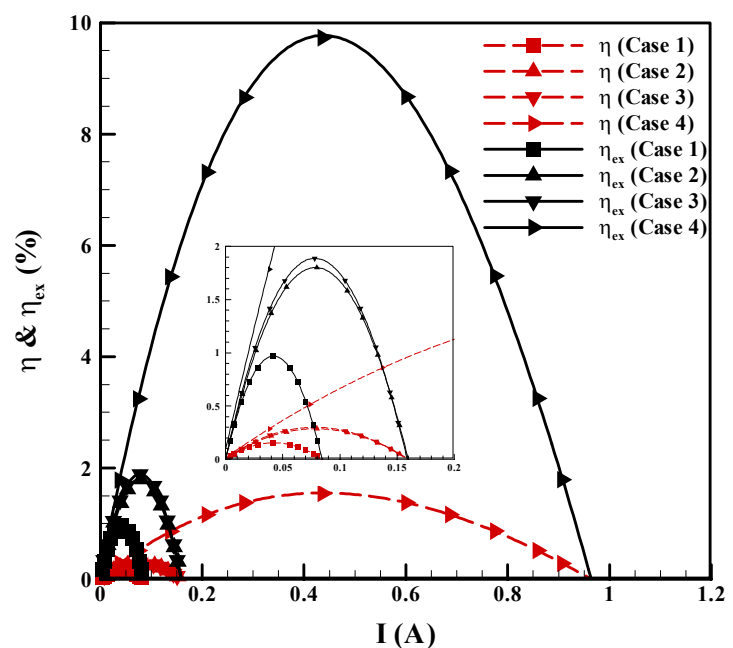

Fig. 4. Effects of heat transfer coefficient on TEG performance. The inset shows the performance in the current range of 0-0.2 A.

Another analysis was conducted in which the heat reservoir temperatures were varied. The analysis focused on the effect of the heat reservoir temperature on TEG performance. Therefore, the heat transfer coefficients at both sides of the TEG were set equal to that of the base case given in Table. 1 . The results of varying the hot-reservoir temperature $T_{H}$ and cold-reservoir temperature $T_{L}$ are given in Fig. 5 and Fig. 6, respectively. Comparing the curve of $T_{H}=70{ }^{\circ} \mathrm{C}$ with that of $T_{H}=90{ }^{\circ} \mathrm{C}$ shown in Fig. 5 (a), the results show that the maximum $\eta$ is increased by $40.2 \%$ but the maximum $\eta_{e x}$ is only increased by $3.45 \%$. The reason is that at the current corresponding to maximum $\eta_{e x}$, the exergy input increase significantly with increasing the hot-reservoir temperature, as shown in Fig. 5 (b). Therefore, the maximum conversion ratio of energy quality is only enhanced slightly when the hot-reservoir temperature increases even though the maximum conversion ratio of energy quantity is improved greatly. Furthermore, it is worth noting that when a lower hot-reservoir temperature than that of the base case is employed (i.e. $T_{H}=70{ }^{\circ} \mathrm{C}$ ), a higher $\eta_{e x}$ can be obtained for a small current (for example, $I=0.05 \mathrm{~A}$ ). However, $\eta$ does not exhibit this trend; it is always improved with the increasing hot-reservoir temperature in the considered range of electrical current. One explanation for this is that when hot-reservoir temperature increases, the major exergy output (the power produced by Seebeck effect $\left.\alpha I\left(T_{H}-T_{L}\right)^{(17)}\right)$ is improved slightly for a small current whereas the exergy input is improved significantly (see Fig 5(b)). Therefore, the results reveal that exergy analysis may be more suitable than energy analysis for evaluating the potential of low-temperature waste heat recovery using a TEG.

The above-mentioned trend can also be found in Fig. 6. However, there is almost no change in the maximum $\eta_{e x}$, whereas the maximum $\eta$ increases when the cold reservoir temperature decreases. If the heat transfer coefficients at the hot side and cold side of the TEG are switched, the maximum $\eta_{e x}$ increases with decreasing cold-reservoir temperature, as shown in Fig. 7. The results clearly indicate that the effect of cold-reservoir temperature on TEG performance is related to the heat transfer coefficients at both sides of the TEG. Under the operating conditions of the base case, increasing the hot-reservoir temperature is a better approach than decreasing the cold-reservoir temperature for improving the maximum $\eta_{e x}$. 

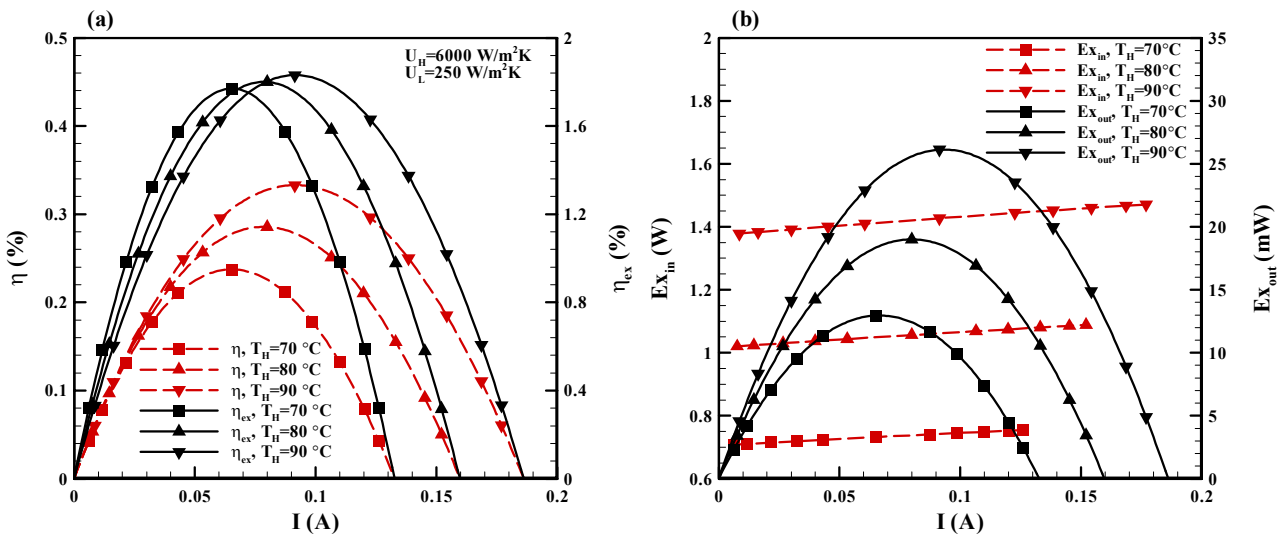

Fig. 5. Effects of hot-reservoir temperature on TEG performance in terms of (a) energy and exergy efficiency and (b) exergy input and exergy output.

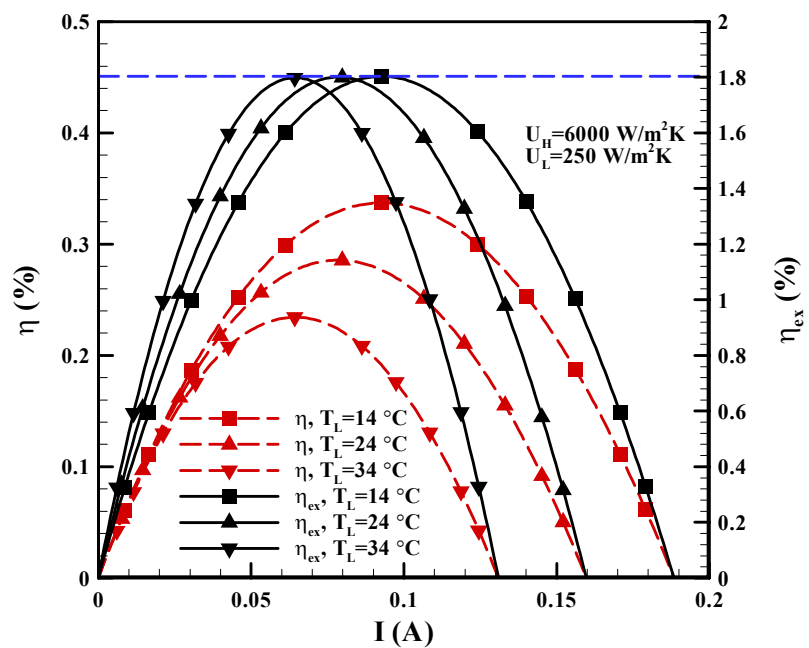

Fig. 6. Effects of cold-reservoir temperature on TEG performance.

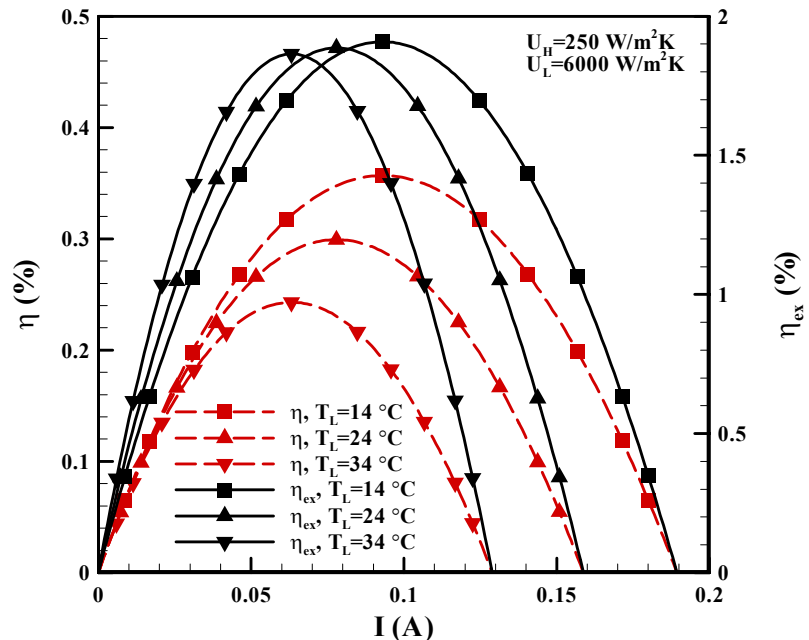

Fig. 7. Effects of cold-reservoir temperature on TEG performance at the conditions of $U_{H}=250 \quad \mathrm{Wm}^{-2} \mathrm{~K}$ and $U_{L}=6000 \mathrm{Wm}^{-2} \mathrm{~K}$.

\subsection{Influence of internal irreversibilities}

The temperature-dependent material properties were considered to investigate the influence of internal irreversibilities on TEG performance. The material properties of the 
TEG are given in Eqs. (16) (18) and the temperature dependence curves are shown in Fig. 8. Based on the above discussion, the hot-reservoir temperature was regarded as the parameter with the other operating conditions fixed (see Table 1). The results of the temperature-dependent Seebeck coefficient, thermal conductivity, and electrical resistivity are compared with the constant properties obtained at $27^{\circ} \mathrm{C}$ in Fig. 9, Fig. 10, and Fig. 11, respectively.
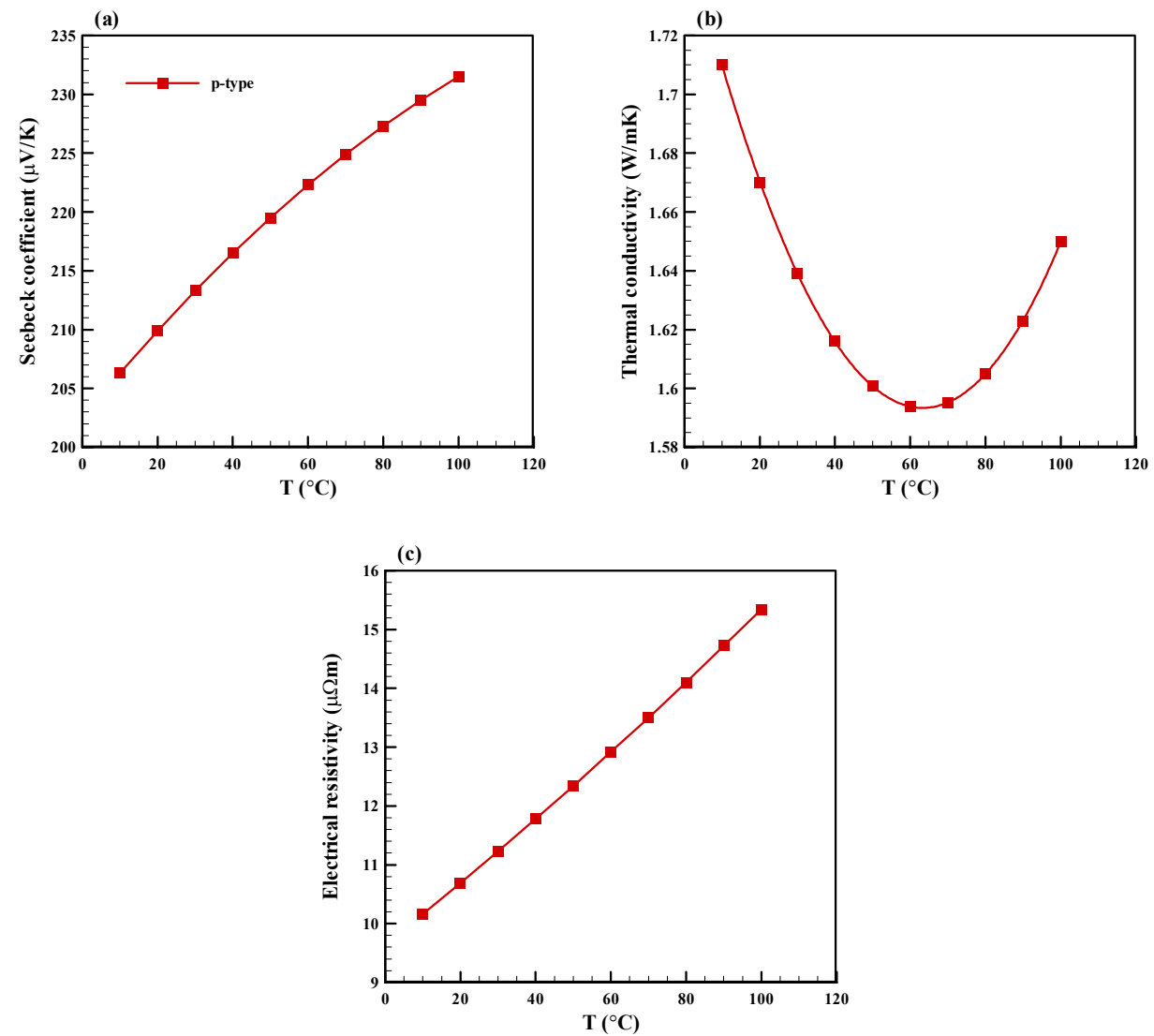

Fig. 8. Temperature-dependent curves of (a) Seebeck coefficient, (b) thermal conductivity, and (c) electrical resistivity.

It is well-known that the temperature-dependent Seebeck coefficient causes the Thomson effect. This effect has been demonstrated that it can improve the performance of the thermoelectric cooler ${ }^{(33)}$. The performance of TEG with Thomson effect are illustrated in Fig. 9. This figure indicates that both $\eta$ and $\eta_{e x}$ values improve with different hot-reservoir temperature. The improvements of the maximum $\eta$ and maximum $\eta_{e x}$ are the same, reaching $7.84 \%$ compared to the case with constant properties obtained at $27^{\circ} \mathrm{C}$, when the hot reservoir temperature of $90{ }^{\circ} \mathrm{C}$ is used. As shown in Fig. 10, the TEG performance is improved when the temperature-dependent thermal conductivity is considered. However, the improvement in performance is smaller than that obtained for the case with temperature-dependent Seebeck coefficient. One explanation for this is that the thermal conductivity increases when the temperature is above $63{ }^{\circ} \mathrm{C}$, as shown in Fig. 8 (b), and thus enhances the irreversible effect. 

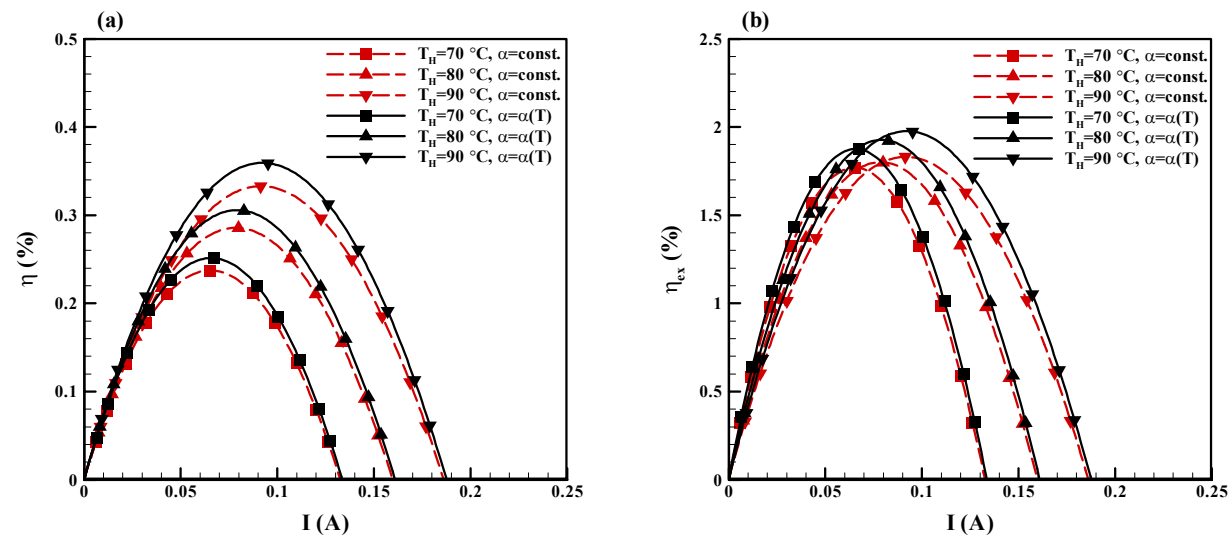

Fig. 9. TEG performance with temperature-dependent Seebeck coefficient under various hot-reservoir temperatures. (a) Energy efficiency and (b) exergy efficiency.
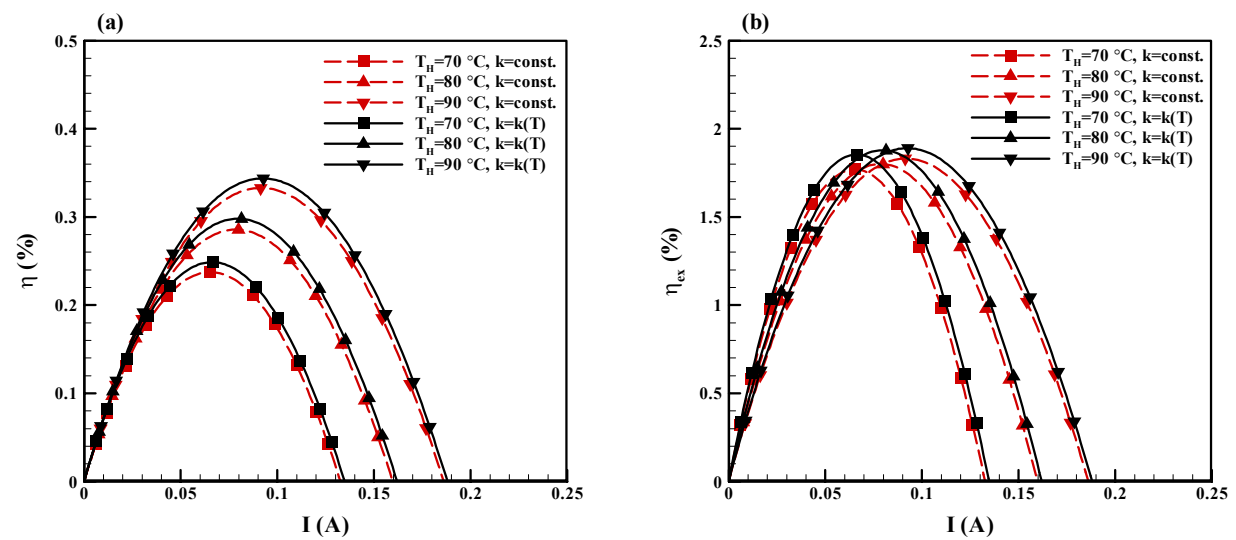

Fig. 10. TEG performance with temperature-dependent thermal conductivity under various hot-reservoir temperatures. (a) Energy efficiency and (b) exergy efficiency.

When the temperature-dependent electrical resistivity is considered, as shown in Fig. 11 (a), even though $\eta$ still improves with increasing hot-reservoir temperature, a lower $\eta$ value is obtained compared to that obtained with constant material properties. Especially under the condition of $T_{H}=90{ }^{\circ} \mathrm{C}$, the maximum $\eta$ is reduced by $14.34 \%$ compared to that with constant material properties. As a result, the maximum $\eta_{e x}$ decreases slightly when the hot-reservoir temperature increases, as shown in Fig. 11 (b). That is, the maximum conversion of energy quality decreases when the hot-reservoir temperature increases, which cannot be found by energy analysis. This phenomenon is quite different from the results of the other two temperature-dependent material properties, where both maximum $\eta$ and $\eta_{e x}$ increase with increasing hot-reservoir temperature. These results reveal that, for a practical TEG system, the temperature dependence of the Seebeck coefficient and electrical resistivity plays an important role in TEG performance, especially for electrical resistivity. 

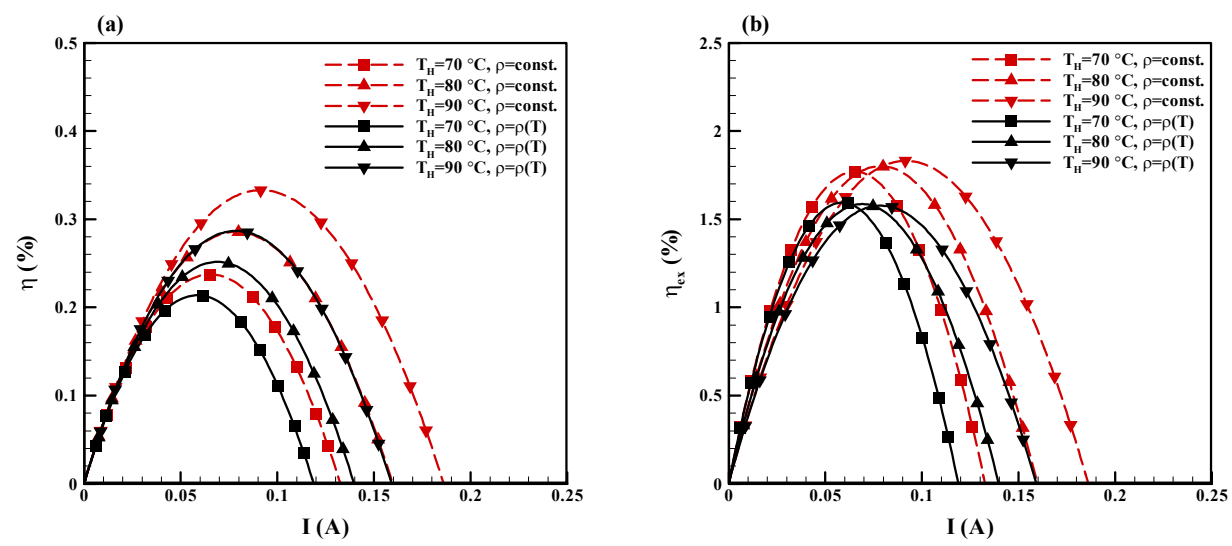

Fig. 11. TEG performance with temperature-dependent electrical resistivity under various hot-reservoir temperatures. (a) Energy efficiency and (b) exergy efficiency.

\section{Conclusion}

A TEG system with multi-irreversibilities was investigated using a numerical method. Based on an exergy analysis, two major indexes, namely the energy efficiency and exergy efficiency, were used to evaluate TEG performance. The results show that the existence of heat loss results in slight reductions of maximum energy and exergy efficiencies. However, the case with heat loss can still provide better energy and exergy efficiencies than those obtained without heat loss for a large electrical current. The performance indexes increase with increasing heat transfer coefficients at the hot side and cold side. Noticeable enhancements in the performance indexes were observed only when the heat transfer coefficients of both sides were increased simultaneously.

The results indicate that for a small electrical current, a higher exergy efficiency than that of the base case can be obtained using a lower hot-reservoir temperature or a higher cold-reservoir temperature, but at the expense of lower energy efficiency. Additionally, the maximum exergy efficiency can be improved by increasing the hot-reservoir temperature. However, the cold-reservoir temperature does not affect the maximum exergy efficiency under the conditions of the base case. Therefore, this study suggests that increasing the hot-reservoir temperature is a better approach to enhancing TEG performance. If the Seebeck coefficient or thermal conductivity is temperature-dependent, the TEG performance is better than that with constant properties obtained at $T=27^{\circ} \mathrm{C}$. However, the case with constant properties performs better than that with temperature-dependent electrical resistivity. Although the energy efficiency can still be enhanced by increasing the hot-reservoir temperature, the maximum exergy efficiency decreases. For a practical TEG system, this situation should be taken into account.

\section{Acknowledgements}

The authors acknowledge the financial support by the National Science Council, Taiwan, ROC, on this research. The authors are also grateful to Professor Wei-Hsin Chen at National University of Tainan, for valuable discussion and suggestions regarding this paper.

\section{References}

(1) Rowe D.M., Thermoelectric waste heat recovery as a renewable energy source, International Journal of Innovations in Energy Systems and Power, Vol.1, No.1 (2006), pp.13-23. 
(2) Noie-Baghban S.H. and Majideian G.R., Waste heat recovery using heat pipe heat exchanger (HPHE) for surgery rooms in hospitals, Applied Thermal Engineering, Vol.20 (2000), pp.1271-1282.

(3) Chen W.H. and Chen J.C., Combustion characteristics and energy recovery of a small mass burn incinerator, International Communications in Heat and Mass Transfer, Vol.28, No.3 (2001), pp.299-310.

(4) Chen W.H., Chung Y.C. and Liu J.L., Analysis on energy consumption and performance of reheating furnaces in a hot strip mill, International Communications in Heat and Mass Transfer, Vol.32 (2005), pp.695-706.

(5) Shih H.Y. and Huang Y.C., Thermal design and model analysis of the Swiss-roll recuperator for an innovative micro gas turbine, Applied Thermal Engineering, Vol.29 (2009), pp.1493-1499.

(6) Chen W.H., Chiu T.W. and Hung C.I., Enhancement effect of heat recovery on hydrogen production from catalytic partial oxidation of methane, International Journal of Hydrogen Energy, Vol.35 (2010), pp.7427-7440.

(7) Chen W.H. and Syu Y.J., Thermal behavior and hydrogen production of methanol steam reforming and autothermal reforming with spiral preheating, International Journal of Hydrogen Energy, Vol.36 (2011), pp.3397-3408.

(8) Gou X., Xiao H. and Yang S., Modeling, experimental study and optimization on low-temperature waste heat thermoelectric generator system, Applied Energy, Vol.87 (2010), pp.3131-3136.

(9) Hsu C.T., Huang G.Y., Chu H.S., Yu B. and Yao D.J., Experiments and simulations on low-temperature waste heat harvesting system by thermoelectric power generators, Applied Energy, Vol.88 (2011), pp.1291-1297.

(10) Bell L.E., Cooling, heating, generating power, and recovering waste heat with thermoelectric systems, Science, Vol.321, No.5895 (2008), pp.1457-1461.

(11) Chen M., Lund H., Rosendahl L.A. and Condra T.J., Energy efficiency analysis and impact evaluation of the application of thermoelectric power cycle to today's CHP systems, Applied Energy, Vol.87 (2010), pp.1231-1238.

(12) Hodes M., Optimal pellet geometries for thermoelectric power generation, IEEE Transactions on Components and Packaging Technology, Vol.33, No.2 (2010), pp.307-318.

(13) Maneewan S. and Chindaruksa S., Thermoelectric power generation system using waste heat from biomass drying, Journal of Electronic Materials, Vol.38 (2009), pp.974-980.

(14) Hsiao Y.Y., Chang W.C. and Chen S.L., A mathematic model of thermoelectric module with applications on waste heat recovery from automobile engine, Energy, Vol.35 (2010), pp.1447-1454.

(15) Jang B., Han S. and Kim J.Y., Optimal design for micro-thermoelectric generators using finite element analysis, Microelectronic Engineering, Vol.88 (2011), pp.775-778.

(16) Wang C.C., Hung C.I. and Chen W.H., Design of heat sink for improving the performance of thermoelectric generator using two-stage optimization, Energy, Vol.39 (2012), pp.236-245.

(17) Wu C., Analysis of waste-heat thermoelectric power generators, Applied Thermal Engineering, Vol.16 (1996), pp.63-69.

(18) Chen J., The maximum power output and maximum efficiency of an irreversible Carnot heat engine, Journal of Physics D: Applied Physics, Vol.27, No.6 (1994), pp.1144-1149.

(19) Chen J. and Wu C., Analysis on the performance of a thermoelectric generator, Journal of Energy Resources Technology-Trans ASME, Vol.122 (2000), pp.61-63. 
(20) Niu X., Yu J. and Wang S., Experimental study on low-temperature waste heat thermoelectric generator, Journal of Power Sources, Vol.188 (2009), pp.621-626.

(21) Chen M., Rosendahl L.A. and Condra T., A three-dimensional numerical model of thermoelectric generators in fluid power systems, International Journal of Heat and Mass Transfer, Vol.54 (2011), pp.345-355.

(22) Dincer I. and Rosen M.A., Exergy, energy, environment and sustainable development, (2007), 1st ed., Oxford, UK, Elsevier.

(23) Orhan M.F., Dincer I. and Rosen M.A., An exergy-cost-energy-mass analysis of a hybrid copper-chlorine thermochemical cycle for hydrogen production, International Journal of Hydrogen Energy, Vol.35 (2010), pp.4831-4838.

(24) Rakopoulos C.D. and Giakoumis E.G., Second-law analyses applied to internal combustion engines operation, Progress in Energy and Combustion Science, Vol.32 (2006), pp.2-47.

(25) Tyagi S.K., Wang S., Singhal M.K., Kaushik S.C. and Park S.R., Exergy analysis and parametric study of concentrating type solar collectors, International Journal of Thermal Sciences, Vol.46 (2007), pp.1304-1310.

(26) Wang J., Dai Y. and Gao L., Exergy analyses and parametric optimizations for different cogeneration power plants in cement industry, Applied Energy, Vol.86 (2009), pp.941-948.

(27) Ozgener L. and Ozgener O., Monitoring of energy exergy efficiencies and exergoeconmic parameters of geothermal district heating systems (GDHSs), Applied Energy, Vol.86 (2009), pp.1704-1711.

(28) Barelli L., Bidini G., Gallorini F. and Ottaviano A., An energetic-exergetic analysis of a residential CHP system based on PEM fuel cell, Applied Energy, Vol. 88 (2011), pp.4334-4342.

(29) Sharp J., Bierschenk J. and Lyon Jr H.B., Overview of solid-state thermoelectric refrigerators and possible applications to on-chip thermal management, Proceedings of the IEEE, (2006-8), pp.1602-1612.

(30) Eswararmoorthy M. and Shanmugam S., Thermodynamic analysis of solar parabolic dish thermoelectric generator, International Journal of Renewable Energy Technology, Vol.1, No.3 (2010), pp.348-360.

(31) Silvester P.P. and Ferrari R.L., Finite elements for electrical engineers, (1996), 3rd ed. New York: Cambridge University Press.

(32) Ahiska R. and Ahiska K., New method for investigation of parameters of real thermoelectric modules, Energy Conversion and Management, Vol.51 (2010), pp.338-345.

(33) Chen W.H., Liao C.Y. and Hung C.I., A numerical study on the performance of miniature thermoelectric cooler affected by Thomson effect, Applied Energy, Vol.89 (2012), pp.464-473. 